\section{Salud sexual y reproductiva en tiempos del COVID-19: accesibilidad de mujeres sordas y con discapacidad auditiva}

\author{
BIELKA CARVAJAL G. ${ }^{1, a}$, JAME REBOLLEDO S. ${ }^{2, b}$, \\ NINOSKA FLÁNDEZ ${ }^{3, \mathrm{c}}$, TANIA FARIÑA ${ }^{3, \mathrm{c}}$, VIANNEY SIERRALTA ${ }^{4, \mathrm{~d}}$
}

\section{Access of chilean deaf women to healthcare information and reproductive care}

Background: The COVID-19 pandemic threatened the accessibility and response of healthcare systems worldwide. People with disabilities face specific access challenges to healthcare services and to healthcare information in accessible formats. Aim: To explore how deaf women acceded to information and sexual and reproductive health care during the first wave of COVID-19. Material and Methods: Sixty-one women with a median age of 32 years diagnosed with deafness and hearing loss were surveyed using an online questionnaire about access to healthcare information and midwifery care during the COVID-19 pandemic. Results: Forty-nine percent of respondents lived in the Metropolitan Region. Sixty-eight percent of respondents mentioned that information about COVID-19 was not accessible for them. The main source of information that they used to learn about the pandemic were videos made by deaf people available on the internet. Seventy-five percent of women reported that they had not received accessible information about sexual and reproductive health, and $70.0 \%$ of women requiring midwife care could not book an appointment. Conclusions: The pandemic generated a crisis in the Chilean healthcare system that demands a new strategy to ensure people's healthcare access. People with disabilities such as those herein studied are marginalized when these new policies are being discussed and implemented. Decision-makers and sexual and reproductive health services must improve their strategies to allow women with disabilities, particularly deaf women gain access.

(Rev Med Chile 2021; 149: 1317-1321)

Key words: COVID-19; Chile; Health Services Accessibility; Midwifery; Persons With Hearing Impairments.
'Departamento de Promoción de la Salud de la Mujer y el Recién Nacido. Universidad de Chile.

Santiago, Chile.

2Departamento de Kinesiología. Universidad de Chile. Santiago,

Chile.

${ }^{3}$ Escuela de Obstetricia y

Puericultura. Universidad de

Chile. Santiago, Chile.

${ }^{4}$ Fundación Nellie Zabel.

aMatrona, Magíster Salud

Pública. Estudiante de Doctorado,

Universidad de Mánchester.

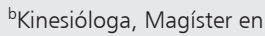

Psicología Comunitaria.

'Licenciada en Obstetricia y

Puericultura.

${ }^{d}$ Abogada, Presidenta de la

Fundación Nellie Zabel.

Financiamiento: Esta investigación no contó con una fuente de financiamiento.

Recibido el 30 de octubre de 2020, aceptado el 1 de julio de 2021.

Correspondencia a:

Bielka Carvajal Gutiérrez Av. Independencia 1027, Independencia. Santiago, Chile. bielkacarvajal@uchile.cl

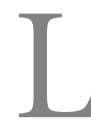

a situación provocada por el COVID-19 ha puesto a prueba al sistema de salud y la organización social en su conjunto. En la región de las Américas, se han interrumpido parcialmente los servicios de salud ambulatoria ${ }^{1}$, lo que en Chile se puede visualizar a través de la disminución del número de consultas en Servicios de Urgencia por patologías no directamente relacionadas al COVID- $19^{2}$.
Las personas con discapacidad tienen dificultades específicas para acceder a los servicios, entre ellas, el requerimiento de contar de información en formato accesible ${ }^{3}$. En particular, la comunicación a través de lengua de señas es crítica para que el mensaje entregado llegue a una porción importante de la comunidad Sorda, quienes de acuerdo con la literatura, presentan menores índices de alfabetización en salud, poco acceso a prevención y 
mayores barreras en la comunicación para acceder a servicios sanitarios que la población general ${ }^{4-6}$. En esta materia, el Comité de Naciones Unidas sobre los Derechos de las Personas con Discapacidad exhortó a las autoridades internacionales a tomar medidas para responder adecuadamente durante la pandemia, que aseguren la inclusión y la participación efectiva de las personas con discapacidad, es decir, no permitir la discriminación ${ }^{7}$.

Si bien estudios en Brasil, sugieren que la comunidad Sorda visita con mayor frecuencia las consultas médicas generales, las mujeres Sordas registran menos controles ginecológicos que la población general ${ }^{8}$. Debiendo enfrentar barreras relacionadas con la comunicación, costos y discriminación ${ }^{5}$. En Chile, el Ministerio de Salud en un esfuerzo por disminuir las barreras en la comunicación, implementó en junio de 2020 un sistema de consulta en lengua de señas chilena (LSCh) ${ }^{9}$. Servicio que si bien llega tarde en términos preventivos del COVID-19 permite acercar la información en salud a este grupo de la población.

El comprender la discapacidad como el resultado de la interacción entre las personas con alguna deficiencia y las barreras contextuales, permite visualizar la existencia de barreras como impedimentos para ejercer los derechos humanos individuales, incluido el acceso a salud y en específico el acceso a salud sexual y reproductiva ${ }^{10}$. Esta comunicación busca describir el acceso a información y atención en salud sexual y reproductiva por parte de mujeres de la comunidad Sorda durante la primera ola de COVID-19.

\section{Material y Método}

Estudio exploratorio, transversal y descriptivo. Este artículo es parte de una investigación mayor sobre el acceso a atención con matrona/ón de mujeres Sordas y con discapacidad auditiva que viven en Chile. Se consideraron elegibles a aquellas mujeres adultas que se autodefinieron como personas Sordas y/o tenían un diagnóstico médico de hipoacusia y que vivieran en el territorio nacional durante la realización de la encuesta. Debido a la escasa información disponible sobre el número de personas Sordas y con hipoacusia en el país y la dificultad para contactarlas, este estudio no se propuso un tamaño muestral a priori.

\section{Muestreo}

El muestreo de este estudio fue no aleatorio, utilizando un formulario electrónico el cual se difundió en las redes sociales de organizaciones de personas Sordas y con discapacidad auditiva. El enlace estuvo disponible por 5 semanas desde el 21 de julio del año 2020. Todas las personas que recibieron el hipervínculo podían compartir el mismo. Para definir el término del período de reclutamiento, se esperó a no recibir respuesta por al menos 10 días corridos, a pesar de la difusión del proyecto por diferentes canales y organizaciones de personas Sordas y con discapacidad auditiva.

\section{Instrumento}

Se diseñó una encuesta sobre el acceso de mujeres Sordas y con discapacidad auditiva a atención con matrona/ón. Esta encuesta fue validada por personas de la comunidad Sorda y estuvo disponible en castellano escrito y en LSCh. En ella hubo una sección dedicada al impacto del COVID-19 en dicho acceso, siendo la respuesta a estas preguntas el objetivo de esta comunicación.

\section{Aspectos éticos}

Esta investigación contó con la aprobación del comité de ética en investigación en seres humanos de la Facultad de Medicina de la Universidad de Chile, registro 129-2019. Todas las personas que participaron en el estudio dieron su consentimiento electrónico al inicio de la encuesta. El consentimiento estuvo disponible de manera escrita y en LSCh para asegurar su máxima comprensión.

\section{Resultados}

Durante las 5 semanas en que la encuesta estuvo disponible, 63 personas abrieron el vínculo, 2 $(3,1 \%)$ de ellas declinando continuar con la misma luego de leer/ver el consentimiento informado. La mediana de edad de las participantes fue de 32 años (RIC 7). Las participantes pertenecieron a 10 regiones del país, predominando aquellas de la Región Metropolitana (49,2\%). De las 61 participantes, $68,9 \%$ se reconoció como persona Sorda, 6,6\% como persona con discapacidad auditiva y $24,6 \%$ en ambas categorías. El $81,9 \%$ de las encuestadas describió que su diagnóstico de salud correspondía a una hipoacusia severa o profunda, $9,8 \%$ a una hipoacusia moderada y $8,2 \%$ a 
una hipoacusia leve. La forma de comunicación preferente para estas mujeres fue mediante LSCh (47,5\%), seguida de 'oral' (21,3\%), 'escrita' $(9,8 \%)$ y 'LSCh y oral' (9,8\%).

En relación con el acceso a la información, 57 de $61(93,4 \%)$ mujeres respondieron este apartado. De ellas, un 68,4\% $(\mathrm{n}=39)$ menciona que la información sobre la pandemia causada por el COVID-19 'No' ha sido accesible. Este porcentaje aumenta a $75,4 \%(n=43)$ cuando se consulta por acceso a información sobre salud sexual y reproductiva específicamente. Respecto a las fuentes de información sobre COVID-19, la mayoría de estas mujeres la obtiene a través de vídeos grabados por personas Sordas; seguido por internet o vídeos grabados por personas oyentes con intérprete de lengua de señas (ILS). En cuanto a información sobre salud sexual y reproductiva, las fuentes de información preferentes fueron los prestadores de salud; seguido por vídeos de personas oyentes con intérprete y familiares; amigos y/o pareja (Tabla 1). Finalmente, al consultar si ha podido acceder a atención con matrona/ón durante la pandemia, $70,0 \%$ de las mujeres que requirieron atención no pudieron acceder a la misma (Tabla 2).

Tabla 1. Accesibilidad a la información en salud durante el período de marzo a julio del año 2020

\begin{tabular}{|c|c|c|}
\hline & $\mathbf{n}$ & $\%$ \\
\hline \multicolumn{3}{|c|}{ ¿Considera que ha tenido acceso a información accesible sobre la pandemia? } \\
\hline Sí & 18 & 31,6 \\
\hline No & 39 & 68,4 \\
\hline \multicolumn{3}{|c|}{ Fuente preferente donde obtuvo dicha información } \\
\hline Vídeos grabados por personas sordas & 6 & 33,3 \\
\hline Familiares, amigos y/o pareja & 4 & 22,2 \\
\hline Internet & 2 & 11,1 \\
\hline Vídeos de personas oyentes con intérprete & 2 & 11,1 \\
\hline Redes sociales & 1 & 5,6 \\
\hline Prestador de salud & 1 & 5,6 \\
\hline Televisión & 1 & 5,6 \\
\hline Otra & 1 & 5,6 \\
\hline \multicolumn{3}{|c|}{ ¿Considera que ha tenido acceso a información accesible sobre salud sexual y reproductiva? } \\
\hline Sí & 14 & 24,6 \\
\hline No & 43 & 75,4 \\
\hline \multicolumn{3}{|c|}{ Fuente preferente donde obtuvo dicha información } \\
\hline Vídeos grabados por personas sordas & 1 & 7,1 \\
\hline Familiares, amigos y/o pareja & 3 & 21,4 \\
\hline Internet & 0 & - \\
\hline Vídeos de personas oyentes con intérprete & 3 & 21,4 \\
\hline Redes sociales & 2 & 14,3 \\
\hline Prestador de salud & 4 & 28,6 \\
\hline Televisión & 1 & 7,1 \\
\hline Otra & 0 & - \\
\hline
\end{tabular}

Tabla 2. Demanda de atención por matrona/ón durante el período de marzo a julio del año 2020

\begin{tabular}{|l|c|}
\hline ¿Ha requerido atención por matrona o matrón durante la pandemia? & n \\
\hline No, no he requerido & 24 \\
\hline Sí, he requerido y pude acceder a ella & 42,1 \\
Sí, he requerido pero no pude acceder a ella & 10 \\
\hline
\end{tabular}




\section{Discusión}

En el ámbito de salud sexual y reproductiva (SSR), El Fondo de Población de Naciones Unidas (UNFPA) proyectaba que 47 millones de mujeres en países de bajo y mediano ingreso no podrán acceder a anticonceptivos modernos si la disrupción de los servicios continuaba por más de 6 meses ${ }^{11}$. Este escenario por supuesto tendrá un correlato en el incremento de los embarazos no deseados y será acompañado de un aumento en la violencia de género ${ }^{11}$. Según Naciones Unidas, las personas con discapacidad (en especial las niñas y mujeres) en situaciones normales ya se encuentran marginadas en el acceso a información y a salud sexual y reproductiva, situación que solo se espera empeore producto del COVID-19 ${ }^{12,13}$. De acuerdo con lo observado en esta encuesta, durante la primera ola de la pandemia un porcentaje relevante de estas mujeres no han podido acceder a información ni a atención con matrona/ón quien es la profesional que realiza la mayor cantidad de prestaciones relacionadas con SSR en nuestro país ${ }^{14,15}$. La dificultad en el acceso a este profesional sucede a pesar de que los servicios de SSR son prioritarios en contextos de crisis y se encuentran dentro del paquete de servicios esenciales de la Organización Mundial de la Salud en la respuesta frente al COVID- $19^{16}$.

El brote epidémico provocado por el COVID-19 ha obligado a rediseñar las estrategias para asegurar el acceso a la salud de las personas, disminuyendo el riesgo de contagio tanto para proveedores como para usuarios. No obstante, se debe cuidar que la adición de implementos básicos en el control de la propagación del virus no signifique un aumento en las barreras a grupos que ya veían comprometida su inclusión en dichas esferas ${ }^{17}$. Estrategias como el uso de mascarilla transparente ${ }^{3}$, la implementación de seminarios en línea sobre temáticas de SSR y de teleatención en salud ${ }^{18}$ pueden ayudar a disminuir el aislamiento y mejorar la calidad de atención de SSR de mujeres Sordas y con discapacidad auditiva.

A pesar de su limitado tamaño muestral, este estudio muestra que la propia comunidad de personas Sordas, las redes sociales y los vídeos realizados por personas oyentes apoyados por ILS, son una fuente relevante de información. De ahí la importancia de contar con sitios en internet que provean información accesible y veraz, fortalecer la formación de líderes comunitarios alfabetizados en SSR y la obligatoriedad de que toda información oficial deba estar disponible en formatos accesibles $^{19}$. En esta materia, se debe recordar que comunicarse utilizando la propia lengua, incluida la lengua de señas, así como la disponibilidad de la información, incluida aquella sobre SSR, en formatos accesibles son derechos humanos ${ }^{20}$.

Finalmente, a un año del inicio del brote en nuestro país, con un creciente número de evidencia alertando un empeoramiento en los indicadores de $\mathrm{SSR}^{19}$, las autoras de esta comunicación se preguntan ¿cuándo habrá lineamientos claros a seguir en SSR para disminuir el daño ya causado por la pandemia? y ¿cuánto más haremos esperar a las mujeres en situación de discapacidad para ser consideradas ciudadanas plenas, con pleno acceso a la SSR?

Agradecimientos: Nuestro agradecimiento a las mujeres que colaboran en la Fundación Nellie Zabel por haber participado activamente en la difusión de esta encuesta.

\section{Referencias}

1. Organización Panamericana de la Salud (OPS). Informe de la Evaluación Rápida de la Prestación de Servicios para Enfermedades no Transmisibles Durante la Pandemia de COVID-19 en las Américas [Internet]. 2020 [citado 12 de octubre de 2020]. Disponible en: https:// iris.paho.org/handle/10665.2/52283.

2. Toro L, Parra A, Alvo M, Toro L, Parra A, Alvo M. Epidemia de COVID-19 en Chile: impacto en atenciones de Servicios de Urgencia y Patologías Específicas. Rev Med Chile [Internet]. abril de 2020 [citado 12 de octubre de 2020];148(4):558-60. Disponible en: http://www.scielo.cl/scielo.php?script=sci_arttext\&pi$\mathrm{d}=$ S0034-98872020000400558\&lng=en\&nrm=iso\&tln$\mathrm{g}=\mathrm{en}$.

3. Armitage R, Nellums LB. The COVID-19 response must be disability inclusive. Lancet Public Heal [Internet]. 1 de mayo de 2020 [citado 12 de octubre de 2020];5(5):e257. Disponible en: http://www.ncbi.nlm. nih.gov/pubmed/32224295.

4. Campos V, Cartes-Velásquez R, Campos V, Cartes-Velásquez R. Estado actual de la atención sanitaria de personas con discapacidad auditiva y visual: una revisión breve. Rev Med Chile [Internet]. mayo de 2019 [citado 12 de octubre de 2020];147(5):634-42. Disponible en: http://www.scielo.cl/scielo.php?script=sci_arttext\&pi- 
$\mathrm{d}=$ S0034-98872019000500634\&lng=en\&nrm=iso\&tln$\mathrm{g}=\mathrm{en}$.

5. Kuenburg A, Fellinger P, Fellinger J. Health Care Access Among Deaf People. J Deaf Stud Deaf Educ [Internet]. 1 de enero de 2016 [citado 12 de octubre de 2020];21(1):1-10. Disponible en: https://academic.oup. com/jdsde/article-lookup/doi/10.1093/deafed/env042.

6. Condessa AM, Giordani JM do A, Neves M, Hugo FN, Hilgert JB, Condessa AM, et al. Barreiras e facilitadores à comunicação no atendimento de pessoas com deficiência sensorial na atenção primária à saúde: estudo multinível. Rev Bras Epidemiol [Internet]. 2020 [citado 12 de octubre de 2020];23. Disponible en: http://www.scielo.br/scielo.php?script=sci_arttext\&pi$\mathrm{d}=$ S1415-790X2020000100460\&tlng=pt.

7. Naciones Unidas. Declaración Conjunta: Personas con Discapacidad y COVID-19 [Internet]. 2020 [citado 12 de octubre de 2020]. Disponible en: https://www. un.org/development/desa/disabilities/wp-content/ uploads/sites/15/2020/04/Declaración-Conjunta-Personas-con-Discapacidad-COVID19.pdf.

8. Fuentes-López E, Fuente A. Access to healthcare for deaf people: a model from a middle-income country in Latin America. Rev. Saúde Pública [Internet]. 2020 [cited 2020 Oct 18]; 54: 13. Disponible en: http:// www.scielo.br/scielo.php?script $=$ sci_arttext\&pi$\mathrm{d}=$ S0034-89102020000100211\&lng=en. https://doi. org/10.11606/s1518-8787.2020054001864.

9. Ministerio de Salud. Atención de Salud en Lengua de Señas Chilena [Internet]. Salud Responde. 2020 [citado 12 de octubre de 2020]. Disponible en: https://saludresponde.minsal.cl/atencion-de-salud-en-lengua-de-senaschilena/

10. Lawson A, Beckett AE. The Social and Human Rights Models of Disability: Towards a Complementarity Thesis. Int J Hum Rights [Internet]. 9 de julio de 2020 [citado 12 de octubre de 2020];1-32. Disponible en: https://www.tandfonline.com/doi/full/10.1080/136429 87.2020.1783533.

11. Fondo de Población de las Naciones Unidas. Repercusión de la pandemia de COVID-19 en la planificación familiar y la eliminación de la violencia de género, la mutilación genital femenina y el matrimonio infantil [Internet]. 2020 [citado 11 de octubre de 2020]. Disponible en: https://www.unfpa.org/resources/impact-covid-19-pandemic-family-planning-and-ending-gender-based-violence-female-genital.
12. United Nations. Leaving no one behind: the COVID-19 crisis through the disability and gender lens | United Nations Enable [Internet]. 2020 [citado 11 de octubre de 2020]. Disponible en: https://www.un.org/development/ desa/disabilities/news/dspd/covid-19-disability.html.

13. Fondo de Población de las Naciones Unidas. Mujeres y Jóvenes con Discapacidad [Internet]. 2018 [citado 12 de octubre de 2020]. Disponible en: https://www.unfpa. org/es/publications/mujeres-y-jovenes-con-discapacidad.

14. Ministerio de Salud. Programa de Salud de la Mujer [Internet]. Santiago; 1997 [citado 12 de agosto de 2020]. Disponible en: https://www.minsal.cl/wp-content/ uploads/2015/09/Programa-de-Salud-de-la-Mujer-1997. pdf.

15. Ministerio de Salud, Instituto Chileno de Medicina Reproductiva, Asociación Chilena de Protección de la Familia. Normas Nacionales sobre Regulación de la Fertilidad [Internet]. Santiago: Ministerio de Salud; 2016 [citado 12 de agosto de 2020]. Disponible en: https://www. minsal.cl/wp-content/uploads/2015/09/2018.01.30_ NORMAS-REGULACION-DE-LA-FERTILIDAD.pdf.

16. World Health Organization. Gender and COVID-19 [Internet]. 2020 [citado 11 de octubre de 2020]. Disponible en: https://apps.who.int/iris/handle/10665/332080.

17. Oficina del Alto Comisionado de las Naciones Unidas para los Derechos Humanos. COVID-19 y los Derechos de las Personas con Discapacidad: Directrices [Internet]. 2020 [citado 12 de octubre de 2020]. Disponible en: https:/www.ohchr.org/Documents/Issues/Disability/ COVID-19_and_The_Rights_of_Persons_with_Disabilities_SP.pdf.

18. Grote H, Izagaren F. Covid-19: The communication needs of $\mathrm{D} /$ deaf healthcare workers and patients are being forgotten. BMJ [Internet]. 15 de junio de 2020 [citado 11 de octubre de 2020];369:m2372. Disponible en: http://www.ncbi.nlm.nih.gov/pubmed/32540861.

19. United Nations. A Disability-Inclusive Response to COVID-19 [Internet]. 2020 [citado 12 de octubre de 2020]. Disponible en: https://www.un.org/sites/un2. un.org/files/sg_policy_brief_on_persons_with_disabilities_final.pdf.

20. Asamblea General de Naciones Unidas. Convención sobre los Derechos de las Personas con Discapacidad [Internet]. Ginebra; 2006 [citado 11 de octubre de 2020]. Disponible en: https://www.un.org/esa/socdev/ enable/documents/tccconvs.pdf. 